\title{
Corela
}

Cognition, représentation, langage

$19-2 \mid 2021$

Vol. $19, \mathrm{n}^{\circ} 2$

\section{Morphologie flexionnelle et dérivationnelle en pulaar (peul) du Foûta Tôro}

\section{Oumar Niang}

\section{(2) OpenEdition}

\section{Journals}

Édition électronique

URL : https://journals.openedition.org/corela/13392

DOI : 10.4000/corela.13392

ISSN : $1638-573 \mathrm{X}$

Éditeur

Cercle linguistique du Centre et de I'Ouest - CerLICO

\section{Référence électronique}

Oumar Niang, « Morphologie flexionnelle et dérivationnelle en pulaar (peul) du Foûta Tôro », Corela [En ligne], 19-2 | 2021, mis en ligne le 02 décembre 2021, consulté le 03 janvier 2022. URL : http:// journals.openedition.org/corela/13392; DOI : https://doi.org/10.4000/corela.13392

Ce document a été généré automatiquement le 3 janvier 2022.

\section{(c) (i) (2)(2)}

Corela - cognition, représentation, langage est mis à disposition selon les termes de la licence Creative Commons Attribution - Pas d'Utilisation Commerciale - Partage dans les Mêmes Conditions 4.0 International. 


\title{
Morphologie flexionnelle et dérivationnelle en pulaar (peul) du Foûta Tôro
}

\author{
Oumar Niang
}

\section{Introduction}

1 Dans le cadre de notre description, nous utilisons une morphologie à base de lexème qui analyse les faits en termes de processus (Fradin et Kerleroux, 2003) ${ }^{1}$. Cette morphologie lexématique promeut une approche de type LEXÈME et PARADIGME des faits morphologiques, que nous allons examiner et utiliser dans l'analyse du marqueur de classe en pulaar.

2 Selon cette approche ${ }^{2}$, les deux termes d'une relation morphologique sont identifiés ${ }^{3}$ :

3 - Soit entre un lexème et l'une de ses formes grammaticalisées s'il s'agit de morphologie flexionnelle.

- Soit entre deux lexèmes distincts s'il s'agit de morphologie constructionnelle.

\section{Morphologie flexionnelle}

\subsection{Définition du lexème}

4 Selon Fradin et Kerleroux (2003), le lexème est une unité abstraite, dépourvue de marques flexionnelles. Il est l'instance fondamentale du signe linguistique. Autrement dit, les lexèmes sont dépendants : leur actualisation nécessite donc le recours à des morphèmes comme, par exemple, les marqueurs de classe. Dans le cadre de la morphologie flexionnelle, les «mots grammaticalisés » sont les membres du paradigme d'un lexème particulier. Ainsi, les mots :

waa-ndu " singe ",

baa- $d i$ « singes », 
baa-ngel « petit singe ",

baa-ngal "grand singe ",

mbaa-kon "petits singes",

baa-dee-j-e " grands singes",

5

représentent les « mots grammaticalisés » du lexème WAA "notion de singe » (forme non soumise à la règle d'allomorphie), avec éventuellement un changement de catégorie morphologique lorsqu'on passe d'une classe à une autre.

6 En effet, le lexème WAA, en recevant ${ }^{4}$ :

7 - le marqueur de classe $/ \mathrm{ndu} /$ devient \{nom singe + \{singulier $\}$,

- le marqueur de classe /đi/ devient \{nom singe $+\{$ pluriel\},

- le marqueur de classe /ngel/ devient $\{$ nom singe $\}+\{$ singulier $\}+\left\{\right.$ diminutif ${ }^{5}$,

- le marqueur de classe /ngal/ devient \{nom singe $\}+\{$ singulier $\}+$ augmentatif $\}$,

- le marqueur de classe /kon/ devient $\{$ nom singe $\}+\{$ pluriel $\}+\{$ diminutif $\}$,

- le marqueur de classe /e/ devient \{nom singe $\}+\{$ pluriel $\}+$ augmentatif $\}$.

8 Les formes "grammaticalisées » du lexème WAA peuvent être illustrées de la manière suivante ${ }^{6}$.

\begin{tabular}{|c|c|c|c|}
\hline Lexème & Marqueurs de classe & Mots grammaticalisés & glose \\
\hline WAA & $\begin{array}{l}\text { ndu } \\
\text { di } \\
\text { ngel } \\
\text { ngal } \\
\text { kon } \\
\text { e }\end{array}$ & $\begin{array}{l}\text { waandu } \\
\text { baadi } \\
\text { baangel } \\
\text { baangal } \\
\text { mbaakon } \\
\text { baadeeje }\end{array}$ & $\begin{array}{l}\text { 《 singe » } \\
\text { « singes » } \\
\text { « petit singe » } \\
\text { « grand singe » } \\
\text { « petits singes » } \\
\text { « grands singes » }\end{array}$ \\
\hline
\end{tabular}

9 Ainsi, relativement à la base, les marqueurs de classe : -ndu, - $d i$, -ngel, -ngal, -kon, -e marquent une opération de flexion. De par leur insertion, ils représentent ici le paradigme flexionnel du lexème de base WAA. Les formes instanciées du lexème (waandu, baadi, baangel, baangal, mbaakon, baadeeje) correspondent aux propriétés prises par le marqueur de classe pour ce qui est du nombre (singulier ou pluriel), de la diminutivité et de l'augmentativité (Mohamadou, 1994). Ces mots "grammaticalisés » sont soumis à des variations consonantiques à leur initiale qui transforment d'abord la sonante $w$ en l'occlusive $b$, cette dernière se prénasalise ensuite en $m b$, d'où l'allomorphie constatée dans les formes fléchies : waa, baa, mbaa .

Avec Kerleroux (2003), nous considérons, que dans le cadre de la flexion, les marqueurs de classe sont l'exposant d'une valeur de trait (singulier waa-ndu « singe », pluriel baa$d i$ « singes ») ou d'un ensemble cohérent de valeurs de traits (le singulier du diminutif baa-ngel "petit singe», le singulier de l'augmentatif baa-ngal "grand singe », le pluriel du diminutif mbao-kon " petits singes ", le pluriel de l'augmentatif baa-dee-j-e " grands singes »). C'est ce que nous résumons dans le tableau suivant.

Tableau : Valeurs de traits des marqueurs de classe 


\begin{tabular}{|c|c|c||c|c|}
\hline Marqueurs de classe & \multicolumn{2}{|c||}{ Trait syntaxique } & \multicolumn{2}{c|}{ Trait sémantique } \\
\cline { 2 - 5 } & singulier & pluriel & diminutif & augmentatif \\
\hline ndu & + & & & \\
\hline di & & + & & \\
\hline ngel & + & & + & \\
\hline ngal & + & & & + \\
\hline kon & & + & + & \\
\hline e & & + & & + \\
\hline
\end{tabular}

11 Les «mots grammaticalisés " représentent donc des unités lexicales telles qu'elles apparaissent en emploi dans la langue, c'est-à-dire pourvues des marques correspondant aux catégories fonctionnelles syntaxique et sémantique des marqueurs de classe. En revanche, le lexème est l'unité lexicale considérée hors de tout emploi dans un contexte syntaxique (syntagme, phrase) (Fradin, 2003, p. 80). Dans les langues dotées de flexion, c'est l'unité lexicale non-fléchie, donc sans marqueur de classe en pulaar.

12 À l'instar de Kerleroux (2003, p. 14), nous dirons que le lexème est un type dont les occurrences sont les «mots grammaticalisés ». Ce procédé flexionnel, très productif, permet de structurer en partie le champ lexical des unités nominales du pulaar.

\section{2. avec double affixation}

13 L'affixation des marqueurs de classe peut être réitérée dans certaines unités nominales peu nombreuses sans doute, mais qu'on ne peut négliger. À partir d'une unité lexicale déjà affixée et pourvue d'autonomie lexicale, on peut construire un autre mot par adjonction d'un nouvel affixe.

Exemple:

kos-am « lait »

kos-am-al « peu de lait».

Ces «mots grammaticalisés » sont issus du lexème /HOS/, dont les formes instanciées commencent par kos. L'affixation des marqueurs de classe -am, -al conditionne en effet un radical réalisant à l'initiale l'occlusive non-voisée $k$.

En outre, le processus de réitération affixale est contraint dans la mesure où le singulier du diminutif du mot kos-am-al « un peu de lait» est construit à partir de la forme singulier kos-am « lait », et non à partir du lexème. La présence du marqueur de classe -al a pour effet de supprimer le statut de am en tant que morphème affixal, ce dernier se trouve ainsi absorbé dans le radical, et donc opacifié.

\section{Morphologie constructionnelle}

17 Les procédés morphologiques de dérivation, de redoublement et de composition font partie de la morphologie constructionnelle. À la différence de la dérivation et de la réduplication qui construisent des unités monolexématiques, la composition construit des unités polylexématiques où figurent des lexèmes identifiables par l'analyse. 


\subsection{Morphologie dérivationnelle} pas une classe homogène du point de vue de l'appartenance catégorielle (Kerleroux, 2003) ${ }^{8}$. L'existence de la morphologie dérivationnelle est liée à la nécessité de la différenciation des catégories. En d'autres termes, les lexèmes sont définis comme appartenant à des « catégories majeures » $: \mathrm{N}, \mathrm{V}, \mathrm{ADJ}, \mathrm{ADV}$. En pulaar, le changement de catégorie s'effectue soit par conversion soit par suffixation d'un morphème dérivationnel.

\subsubsection{La dérivation par conversion}

\section{Racine verbale}

jah «notion d'aller»

am «notion de danser»

mah «notion de construire»

yeey «notion de vendre»

substantif déverbal
jah-gol
gam-gol
mah-gol
jeey-gol

glose

«action de marcher»

« action de danser"

" action de construire"

« action de vendre », etc.

21 Le fait que la racine verbale soit convertie directement en un substantif déverbal permet de postuler ici une dérivation avec morphème zéro. Ce procédé morphologique, très productif en pulaar, permet la construction de plusieurs déverbaux à partir d'un même radical. Ainsi, le verbe sow « notion de plier " peut être dérivé en :

$\begin{array}{lll}\text { nom d'action } & \text { cow-gol } & \text { "pliage » } \\ \text { nom avec résultat du procès } & \text { cow-gu } & \text { "pli » } \\ \text { nom locatif } & \text { sow-an-de } & \text { " endroit du pli » } \\ \text { nom d'instrumental } & \text { cow-ir-gal } & \text { "plieur » } \\ \text { nom d'agentif } & \text { cow-oo-wo } & \text { "celui qui plie ». }\end{array}$

10

Par ailleurs, les formes déverbales, obtenues par dérivation, mettent en évidence des constructions dites agentives, qui correspondent en français aux nominaux construits avec le suffixe -eur (vendeur, chanteur, buveur, etc.) Ces agentifs reproduisent les marques d'accord du nom précédent (cf. exemples ci-dessous).

En pulaar, on distingue plusieurs formes d'agentifs selon que le verbe est à la voix active, moyenne ou passive. De sorte que des morphèmes s'ajoutent à la conversion, non pour opérer le transfert de catégorie mais pour ajouter plusieurs valeurs aspectuelles et diathétiques distinctes.

Exemples d'agentifs imparfaits (ou inaccomplis) à la voix active ${ }^{11}$ : 


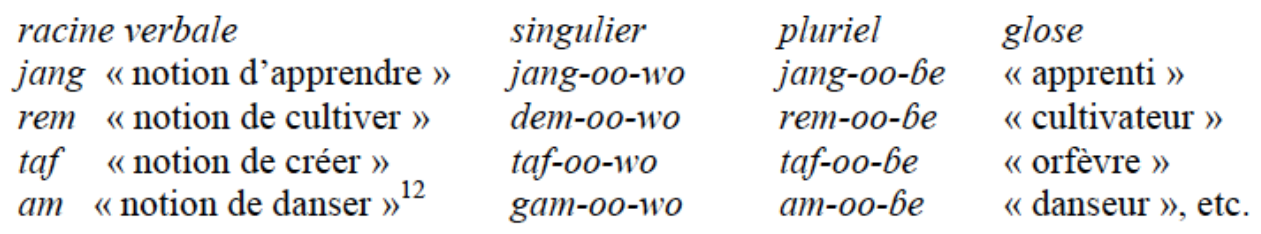

Exemples d'agentifs parfaits (ou accomplis) à la voix active :

$\begin{array}{llll}\text { racine verbale } & \text { singulier } & \text { pluriel } & \text { glose } \\ \text { juul «notion de prier" } & \text { juul-do } & \text { juul-6e } & \text { "croyant» } \\ \text { maay "notion de mourir" } & \text { maay-do } & \text { maay-6e } & \text { "mort» } \\ \text { waas «notion de perdre " } & \text { baas-do } & \text { waas-6e } & \text { "pauvre », etc. }\end{array}$

À la voix moyenne, les agentifs imparfaits (ci-dessous) sont reconnaissables d'abord par la marque aspectuelle verbale $o$, puis par le morphème $t$, ensuite par le morphème d'agentif oo, manifesté après le marqueur de classe.

Exemples d'agentifs imparfaits à la voix moyenne (avec la marque verbale «aa » à l'infinitif):
verbe
jul-aa-de «commercer»
singulier
pluriel
glose
laam-aa-de "gouverner»
jul-o-t-oo-do
jul-o-t-oo-6e
« commerçant »
duw-aa-de «prier»
laam-o-t-oo-do
laam-o-t-oo-be
duw-o-t-oo-do
duw-o-t-oo- $6 e$
« dirigeant »
nyaag-aa-de "quémander »
nyaag-o-t-oo- $d o$
nyaag-o-t-oo- $6 e$
« orant»
« quémandeur », etc.

Quant à l'agentif parfait à la voix moyenne, il est reconnaissable par l'infixe \{ii\} qui apparaît directement après le radical.

Exemples d'agentifs parfaits à la voix moyenne :

$\begin{array}{llll}\text { verbe } & \text { singulier } & \text { pluriel } & \text { glose } \\ \text { hor-aa-de "être inapte à }{ }^{13} & \text { kor-ii-do } & \text { hor-ii-be } & \text { "femme enceinte » } \\ \text { sow-aa-de "plier " } & \text { cow-ii-do } & \text { sow-ii-be } & \text { "femme enceinte » } \\ \text { tund-aa-de "rendre invulnérable " } & \text { tund-ii-do } & \text { tund-ii-be } & \text { "un protégé » } \\ \text { yool-aa-de "se noyer » } & \text { jool-ii-do } & \text { yoo-l-ii-be } & \text { "un noyé », etc. }\end{array}$

13

30 L'agentif à la voix passive est identifiable par le morphème \{aa\} présent dans les exemples suivants, où il apparaît directement après le radical. Contrairement aux agentifs à la voix active ou moyenne, il n'y a pas, au passif, une distinction entre agentifs imparfait et parfait.

31 Exemples d'agentifs à la voix passive (avec la marque verbale « ee » à l'infinitif) :

\begin{tabular}{|c|c|c|c|}
\hline verbe & singulier & pluriel & glose \\
\hline nul-ee-de "être envoyé » & mul-aa-do & mul-aa-be & «un envoyé, prophète \\
\hline haang-ee-de " être fou" & kaang-aa-do & haang-aa-be & «fou » \\
\hline $\begin{array}{l}\text { wamb-ee-de "être porté " } \\
\text { mal-ee-de "être chanceux" }\end{array}$ & bamb-aa-do & $w a m b-a a-b e$ & «troubadour » \\
\hline mal-ee-de « être chanceux » & mal-aa-do & $m a l-a a-b e$ & « chanceux », etc. \\
\hline
\end{tabular}

Nombre d'agentifs se comportent comme des formes lexicalisées tout en impliquant une idée de saillance par rapport au nom de référence. Ainsi, le mot nulaado, issu du verbe nul «commissionner ", réfère à un envoyé précis, c'est-à-dire à un «envoyé de Dieu » qui est le "prophète ». Le mot talloore, formé à partir du verbe tall «rouler», désigne un insecte précis qui est le «bousier». 

permet de transformer en verbes les adjectifs de couleur comme ran «blanc», $6 a$ al " noir ", wod " rouge ", respectivement, en ran-w-u-de "blanchir", bal-w-u-de "noircir », wod- $w$ - $u$-de " rougir ». Mais la majorité des adjectifs deviennent des verbes grâce au morphème suffixal $\{d\}$, comme cela est illustré à travers les exemples suivants :

\begin{tabular}{|c|c|}
\hline Racin & jectivales \\
\hline hes & « neuf $»$ \\
\hline dom & « assoiffé » \\
\hline fam & « rabougri » \\
\hline ool & « jaune » \\
\hline sooy & «brun » \\
\hline dokk & «borgne » \\
\hline tokos & «petit » \\
\hline butt & « gros » \\
\hline
\end{tabular}

14

les formes verbales, on note qu'un radical simple se complexifie par l'insertion de plusieurs morphèmes grammaticaux. Outre le marqueur d'infinitif de, des éléments thématiques tels que les morphèmes itératifs $\{t\}$, causatif $(\{n\}$, comitatif $\{d\}$, locatif $\{r\}$, de réciprocité \{ondir\}, de visée \{oy\} et simulatif $\{k i n\}$ peuvent être attachés à un radical verbal (Sylla, 1982, p. 115-118). C'est ainsi que le verbe yah-de « aller " peut être dérivé en :

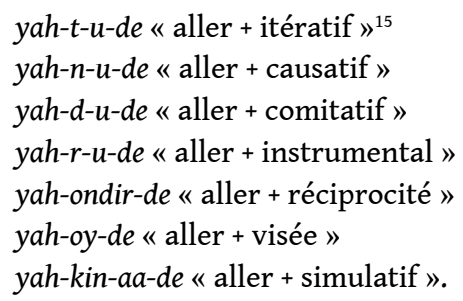

37 Ce procédé morphologique de dérivation par infixation, très productif, participe à la structuration des formes verbales.

\begin{tabular}{|c|c|}
\hline Formes verbales & \\
\hline hes-d-u-de $e^{14}$ & « être neuf » \\
\hline dom-d-u-de & «avoir soif $»$ \\
\hline fam-d-u-de & « être rabougri » \\
\hline$o o l-d-u-d e$ & « devenir jaune » \\
\hline sooy-d-u-de & « devenir brun » \\
\hline dokk-i-d-de & " être borgne" \\
\hline tokos-i-d-de & " être petit» \\
\hline butt-i-d-de & « être gros », etc. \\
\hline
\end{tabular}




\subsection{Composition des unités lexicales} unité nominale com
(Sylla, 1982, p. 195) :

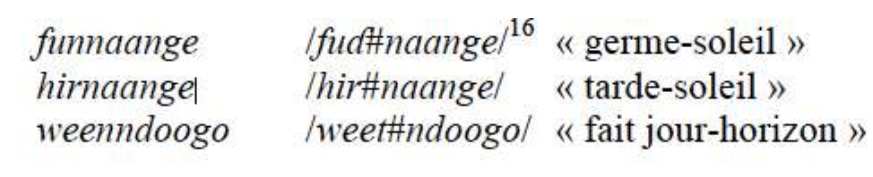
distinguer plusieurs types de composition.

\subsubsection{Composition nominale} mouton $»+$ « Dieu » = « petit insecte rouge $»$.

\subsubsection{Composition verbo-nominale}

En pulaar, la juxtaposition des unités de différentes catégories lexicales permet de

Il n'est pas facile de décider si un enchaînement d'unités lexicales constitue un mot composé ou relève simplement de la combinaison libre d'unités dans le discours. Ainsi holsere nagge " sabot de vache » relève de la combinaison libre dans la mesure où, d'une part, le terme (nagge) peut commuter avec les mots mbaalu "mouton", puccu "cheval », mbeewa "chèvre », ngelooba "chameau », etc. et d'autre part, au terme (holsere), on peut substituer les mots yitere « œil », laaci « queue », nguru " peau ».

En revanche, les éléments composant deftel alla de deftel " petit livre » + alla " Dieu » = "papillon» représentent un enchaînement d'unités lexicales formant un composé. Bien qu'il soit constitué de deux unités morphologiquement différentes, mais lexicalement unique, le composé nominal ne peut commuter les deux places qui le constituent et connaît un figement syntagmatique. Même constat pour baalel alla " petit

Des phénomènes de métaphore peuvent intervenir dans la formation des composés nominaux, puisque le mot deftel, qui signifie littéralement " petit livre », fait allusion au battement d'ailes du papillon qui en s'ouvrant et en se refermant évoque l'image d'un livre, alors que les taches colorées et multiples s'apparentent à une écriture divine (alla). On observe le même glissement sémantique pour le mot baalel alla, dont le premier terme, signifiant littéralement "petit mouton", renvoie à un petit insecte rouge, inoffensif, image généralement attachée au mouton. Ce petit insecte rouge est perçu comme un signe divin qui annonce la saison des pluies, période qui correspond à la renaissance de la vie, pour un peuple vivant de l'élevage et de l'agriculture.

16

A côté de ces exemples, il existe d'autres composés verbo-nominaux avec une structure argumentale où c'est le verbe qui précède toujours le nom. Ce verbe est toujours «transitif » comme le note Sylla (1982) ${ }^{17}$ :
war-jinne
war-hoore
momtu-gondi
ubu-junngel
human binndi
« tueur de diable »
« meurtre»
« sur qui on peut compter»,
" ingratitude »,
« analphabète », etc.

\author{
«Est, levant » \\ « Ouest, couchant» \\ « aube », etc.
}




\subsubsection{Composition avec le morphème de possession jom}

lexématique, permettent d'identifier un lexème de base de ses différentes formes grammaticalisées, lesquelles sont instanciées dans un paradigme flexionnel. Alors que dans le cadre de la dérivation, le changement de catégorie grammaticale, soit par conversion soit par suffixation d'un morphème, aboutit, dans la plupart des cas, à la construction des déverbaux, où la sélection des marqueurs de classes entraîne une allomorphie des radicaux.

\section{BIBLIOGRAPHIE}

Arnott, D. W. (1970), The Nominal and Verbal Systems of Fula, Oxford, Clarendon Press.

Corbin, D. (1987), Morphologie dérivationnelle et structuration du lexique, Lille, Presses Universitaires de Lille.

Fradin, B. et Kerleroux, F. (2003), Quoi de neuf en morphologie ? (introduction), Langages $n^{\circ} 152$, Paris, Larousse, p. 3-11.

Fradin, B. (2003), Nouvelles approches en morphologie, Paris, P.U.F.

Gardes-Tamine, J. (2002), La grammaire: Phonologie, morphologie, lexicologie (3 ${ }^{\text {ème }}$ édition), Paris, Armand Colin, coll. "Cursus".

Huot, H. (2001), Morphologie : forme et sens des mots en français, Paris, Armand Colin.

Kerleroux, F. (2003), « Morpho-logie : la forme et l'intelligible », Langages, n 152, Paris, Larousse, p. 12-32.

Corela, 19-2 | 2021 
Labatut, R. (1973), Le parler d'un groupe de peuls nomades, Nord Cameroun, Paris : Société d'Études linguistiques et Anthropologiques de France, Selaf-Paris.

Lacroix, P. F. (1968), « Le peul », Le langage, Encyclopédie de la Pléaide, Paris.

Lehmann, A. et Martin-Berthet, F. (1998), Introduction à la lexicologie : sémantique et morphologie, Paris, Dunod.

Mel'cuk, I. et Bakiza, E. (1987), « Les classes nominales en kirundi », Paris, Bulletin de la Société de Linguistique de Paris, tome LXXXII, p. 283-341.

Mohamadou, A. (2014), Le verbe en peul. Formes et valeurs en pulaar du Fuuta Tooro, Paris, Karthala.

Mohamadou, A. (1998), « Fonctionnement morphologique et interprétations sémantiques d'un système classificatoire : l'exemple du peul », Faits de langues, $n^{\circ} 11-12$, thématique Les langues d'Afrique sub-saharienne, p. 391-405.

Mohamadou, A. (1994), Classificateurs et représentation des propriétés lexicales en peul (parlers de l'Aadamaawa), Association Linguistique Africaine, Collection « Les Documents de Linguistique Africaine ».

Ndiaye, M. (1983), Morphologie des nominaux et des verbaux du pulaar, Dakar, Centre de linguistique appliquée de Dakar.

Niang, M. (1997), Pulaar-English/English-Pulaar, Standard dictionary, New York, Hippocrene Books.

Niang, O. (2021), « Réanalyse des formes des marqueurs de classe du pulaar (peul) sous l'éclairage de la réduplication », Corela [En ligne], 19-1.

Niang, O. (2007), Description phonologique, morphologique, organisation et fonction de catégorisation des classes nominales en pulaar, thèse de doctorat, MSHS, Université de Poitiers.

Paradis, C. (1986), Phonologie et morphologie : les classes nominales en peul (fula), thèse de doctorat, Université de Montréal.

Sylla, Y. (1982), Grammaire moderne du pulaar, Dakar, Nouvelles Editions Africaines.

Villiard, P. (1993), « Morphologie : tendances actuelles », Tendances actuelles en linguistique générale, sous la direction de Nespoulous, J-L., Neuchâtel, Paris, Delachaux et Niestlé, p. 49-82.

\section{NOTES}

1. Fradin et Kerleroux (2003, p. 6).

2. Selon Fradin (2003, p. 77), la morphologie à base de lexème s'oppose au modèle classique d'analyse structurale qui considère les faits en termes « d'arrangement » d'unités.

3. Kerleroux (2003, p. 19).

4. Toutefois, il convient de souligner que ce n'est pas tout lexème qui peut être fléchi à toutes les formes données dans ces exemples. Les mots pluriel dool-e "forces", ceh-e "cimetières " par exemple n'ont pas de forme fléchie au singulier. Le marqueur de classe oUM n'apparaît qu'avec des notions compactes : kes-um "nouveau», meh-um "rien», etc., non pluralisables et non susceptibles de recevoir les marqueurs du diminutif et de l'augmentatif.

5. Mel'cuk et Bakiza (1987, p. 333-335) analysent en kirundi les affixes du diminutif et de l'augmentatif comme dérivationnels et les marqueurs de classe du singulier et du pluriel comme flexionnels. Or, nous considérons avec Kerleroux, F. (2003, p. 19), que les marqueurs de classe peuvent avoir une «valeur de trait » ou un « ensemble cohérent de valeur de traits » (cf. tableau valeurs de traits des marqueurs de classe). 
6. Niang (2007) et (2021) sur les règles de variations ou d'alternances consonantiques affectant les radicaux nominaux et verbaux du pulaar.

7. Les unités nominales denses font leur diminutif en $K A L$.

8. Kerleroux (2003, p. 21).

9. La présence de ce marqueur dans ces substantifs implique l'idée «d'action » ou de «manière de ».

10. Le passage au locatif, à l'instrumental et à l'agentif nécessite une dérivation avec la présence de morphèmes : $\{n, r, o o\}$ après le radical.

11. Les noms de référence sont ned $d 0$ "personne" au singulier et yimbe " personnes » au pluriel.

12. Jouer d'un instrument de musique.

13. L'état de grossesse véhicule l'image d'un état passif, vécu comme une incapacité à se mouvoir.

14. La distribution des voyelles épenthétiques est liée à la structure syllabique du radical et à la nature des morphèmes. La voyelle $/ \mathrm{u} /$ apparaît dans les radicaux avant le morphème d'infinitif de, alors que /i/ se manifeste dans des radicaux avant le morphème dérivationnel $\{\mathbb{C}\}$.

15. Le même morphème $t$ est également utilisé dans les verbes dits inversifs qui expriment l'idée de renversement. Ces verbes sont toujours à la voix moyenne qui est ici exprimée par la voyelle longue de l'avant-dernière syllabe \{aa\} lorsqu'ils sont au mode infinitif (exemple : humde / hum-t-aa-de « attacher / détacher »).

16. La fusion des deux termes se traduit par l'assimilation régressive de nasalité sur la consonne finale du radical verbal. C'est le même processus phonologique qui affecte la consonne finale de la racine verbale /weet/ « faire jour » au contact de la prénasalisée à l'initiale du mot ndoogu « horizon ».

17. Sylla (1982, p. 195).

\section{RÉSUMÉS}

De manière générale, la formation de mots implique les opérations de dérivation et de composition auxquelles s'attachent les marques de flexion. En pulaar (peul), la flexion, liée à l'affixation des marqueurs de classe et la dérivation, obtenue par conversion ou par suffixation d'un morphème, aboutissent à la formation d'unités monolexématiques, alors que la composition construit des unités polylexématiques.

Generally, word formation involves the derivation and composition operations to which inflection marks are attached. In Pulaar (Fulani), the inflection, linked to the affixation of class markers and the derivation, obtained by conversion or by suffixation of a morpheme, lead to the formation of monolexematic units, while the composition constructs polylexematic units. 
INDEX

Keywords : Fulani, Pulaar, class marker, inflectional morphology, derivational morphology, lexeme, inflectional paradigm, flexion, derivation, conversion, derivational morphem, composition

Mots-clés : peul, pulaar, marqueur de classe, morphologie flexionnelle, morphologie dérivationnelle, lexème, paradigme flexionnel, flexion, dérivation, conversion, morphème de dérivation, composition

\section{AUTEUR}

OUMAR NIANG

Société Linguistique de Paris - Inalco 